\title{
UK launches ambitious tissue/data bank project
}

U K scientists are launching BioBank, an ambitious government-backed population genomics study intended to reveal the combined effects of environment, lifestyle, and genotype on disease risk. The Wellcome Trust and the Medical Research Council (MRC) have each committed an initial $£ 20$ million to the project, with a further $\mathfrak{E} 5$ million coming from the UK Department of Health.

Starting in April next year, the study will recruit half a million UK citizens from 45 to 69 years of age. Plasma and DNA samples will be taken from all subjects, along with details of their exposure to risk-related factors such as dietary factors, alcohol, exercise, pollution, smoking, and even mobilephone use. The study will then track the subjects' subsequent encounters with the National Health Service (NHS), including all medications prescribed and diseases diagnosed, throughout their lives. Researchers hope this will cast light on the causes of cardiovascular, metabolic, musculoskeletal, and neuropsychiatric conditions and cancer. The initial blood samples will define the subjects' initial states of health as well as providing a means of assessing biochemical markers of exposure later on. The 45-69 age group was chosen so that many disease events are likely to occur during the course of the study, providing a continuing stream of data for the next 30 years.

"Linking these [environmental and lifestyle factors and measures of blood pressure and biochemicals] to genetic information will provide a wealth of new information that will not be available elsewhere," says Alan Doyle, the project's scientific program manager at the Wellcome Trust.

The project differs from other large-scale genetic studies principally in that it will prospectively collect detailed questionnaire-based information on environmental factors and lifestyle, says Doyle. Other studies, such as the one in Iceland managed by Reykjavik-based deCODE Genetics (Nat. Biotechnol. 16, 816, 1998) and a public effort planned for Estonia (Nat. Biotechnol. $18,1135,2000)$, are currently retrospective and are subject to concomitant problems of incomplete data and recall bias.

The BioBank project is also expected to be more comprehensive than others because the UK population is descended from several waves of immigration and so is genetically highly heterogeneous. This means it contains many more polymorphisms to investigate than do the relatively

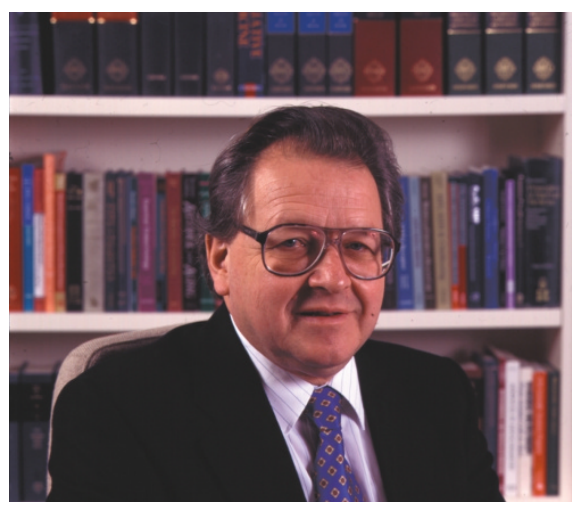

Sir George Radda says the project could lead within 20 years to individualized approaches to disease prevention and treatment.

inbred populations of Iceland and Estonia, for example, although some overlap of results between studies is likely.

But it is the sheer size of the BioBank project that is most significant. There have been other worthwhile studies with a similar aim-such as UK's ALSPAC (Avon Longitudinal Study of Pregnancy and Childhood), which has followed 12,000 families since 1991 to find the genetic and environmental pathways that lead over time to common disorders such as asthma, allergies, and autism. But such studies have generally included too few participants providing biological samples to produce fully consistent and statistically reliable results, according to BioBank's literature.

BioBank's large scale means that it will be well placed to confirm or refute current controversies about the causes of disease. These include the conjectures that cigarette smoking causes ischemic heart disease through its effect on abnormal genes coding for endothelial nitric oxide synthase and apolipoprotein E4, and that eating meat leads to colon cancer through interactions with abnormal genes coding for the enzyme $\mathrm{N}$-acetyltransferase-2. No other type of study (in terms of size and depth) could prove or disprove this kind of theory, according to BioBank.

Even more interesting for the pharmaceutical and biotech industries is that BioBank might cast much light on pharmacogenomics. In particular, it will examine whether an individual's genotype increases the risk of suffering adverse events from certain drugs. Sir George Radda, MRC chief executive, says that the project could lead within 20 years to individualized approach- es to disease prevention and treatment. "Once we understand the genetic bases of various diseases and the genetic differences between individuals that may affect their responses, it may become possible for a [general physician] to prescribe drugs or other treatments designed specifically for people's own genetic makeup."

Despite this, the geneticists involved do not see BioBank as competing with any of the public research projects that are trying to link specific polymorphisms to specific diseases, such as the SNP consortium (Nat. Biotechnol. 17, 526, 1999), or with the private gene discovery studies going on, such as deCODE's successful mapping last October of a gene contributing to lateonset Parkinson's disease in 51 Icelandic families. "Studies looking at families that are highly affected with a particular disease are likely to identify candidate genes faster than our results will be available," says the MRC's Frances Rawle. Instead, BioBank's contribution will be to show the action of known disease genes in an unselected population exposed to random environmental and lifestyle factors. It will also indicate how genes interact with one another. "Private genomics research will, of course, continue," says Doyle, "but is more targeted to specific conditions and does not have the benefit of linkage to NHS records on the scale envisaged in the UK BioBank."

Information obtained from the study will be published, and full details and the raw data will be made available-in an anonymized form-to third parties for legitimate medical research. Any research group will be entitled to apply for access, but proposed study designs will be scrutinized for ethical acceptability before a license is granted. Fees for academic researchers are likely to be much lower than those charged to commercial companies, according to the MRC. "We have always viewed the project as providing an international resource," says Doyle. "There will, however, be safeguards on its use, and access will be strictly controlled."

Meanwhile, some groups criticize BioBank, claiming that it is being driven by commercial interests and profits, whereas others say it might impact on similar research by restricting the amount of grant funds available. And the independent charity GeneWatch UK has demanded a debate in the House of Commons to review the ethical issues and need for legal safeguards. Peter Mitchell, London 